\title{
Part-optimized forming by spatially distributed vaporizing foil actuators
}

\author{
Marlon Hahn ${ }^{1}$ (D) A. Erman Tekkaya ${ }^{1}$ \\ Received: 14 April 2021 / Accepted: 29 June 2021 / Published online: 19 July 2021 \\ (C) The Author(s) 2021
}

\begin{abstract}
Electrically vaporizing foil actuators are employed as an innovative high speed sheet metal forming technology, which has the potential to lower tool costs. To reduce experimental try-outs, a predictive physics-based process design procedure is developed for the first time. It consists of a mathematical optimization utilizing numerical forming simulations followed by analytical computations for the forming-impulse generation through the rapid Joule heating of the foils. The proposed method is demonstrated for an exemplary steel sheet part. The resulting process design provides a part-specific impulse distribution, corresponding parallel actuator geometries, and the pulse generator's charging energy, so that all process parameters are available before the first experiment. The experimental validation is then performed for the example part. Formed parts indicate that the introduced method yields a good starting point for actual testing, as it only requires adjustments in the form of a minor charging energy augmentation. This was expectable due to the conservative nature of the underlying modeling. The part geometry obtained with the most suitable charging energy is finally compared to the target geometry.
\end{abstract}

Keywords Vaporizing foil actuator forming $\cdot$ Impulse distribution $\cdot$ Optimization $\cdot$ Energy deposition $\cdot$ Predictive process design

\section{Introduction}

The use of vaporizing foil actuators (VFA) in manufacturing applications is a rather new approach (for an overview see e.g. Vivek et al. [1]). The majority of research so far focuses on welding through plastic deformation during impact with regard to both experimental [2] as well as modeling [3] findings. Similar to electromagnetic or electrohydraulic forming $[4,5]$, a capacitor bank discharge is used to supply the process energy (Fig. 1 a). But with VFA, the intensive Joule heating due to the discharge current, along with the characteristic voltage curve (Fig. $1 \mathbf{b}$ ), is used to almost instantaneously vaporize distinct regions of thin metal foils. They are typically made of aluminum and serve as disposable actuators. The resulting explosion-like pressure impulse accelerates a workpiece, e.g. a sheet metal blank, from the so-called burst time $\left(t_{b}\right)$ on to form it within around $0.05-1 \mathrm{~ms}$. A reusable pressure-

Marlon Hahn

Marlon.hahn@iul.tu-dortmund.de

1 Institute of Forming Technology and Lightweight Components (IUL), TU Dortmund University, Baroper Str. 303, 44227 Dortmund, Germany transmitting polyurethane layer is often used mostly to avoid arcing between the actuator and the metal workpiece. The work area must occasionally be cleaned from condensed foil matter afterwards.

The application of this technology to forming $(\mathrm{VFA} F)$ is covered in the patent of Vivek et al. [6]. In another patent, Vivek et al. [7] give an idea how to automate the process by supplying the actuators in the form of a consumable tape coming from an industrial coil system.

Regarding the forming behaviour, Cai [8] in his dissertation and related publications investigated the influence of basic process parameters, like polyurethane thickness ( $3 \mathrm{~mm}$ was found to be ideal), foil dimensions, and charging energy on the bulging result of some aluminum sheets experimentally by trial-and-error. He also introduced equations to describe the process to some extent. However, as his approach was based on the so-called electrical Gurney model, he indirectly neglects the plasticity of the workpiece to determine its velocity. And this is not permissible for many forming parts. The same author also showed that one actuator can be used to form two parts at the same time. This was done by either placing two sheets above and below the actuator, or by including two vaporization regions within one actuator in series. 
Fig. 1 a) Principle of vaporizing foil actuator technology, b) exemplary smoothed discharge curve extracts a) Actuator vaporization (expansion) Actuator image after experiment

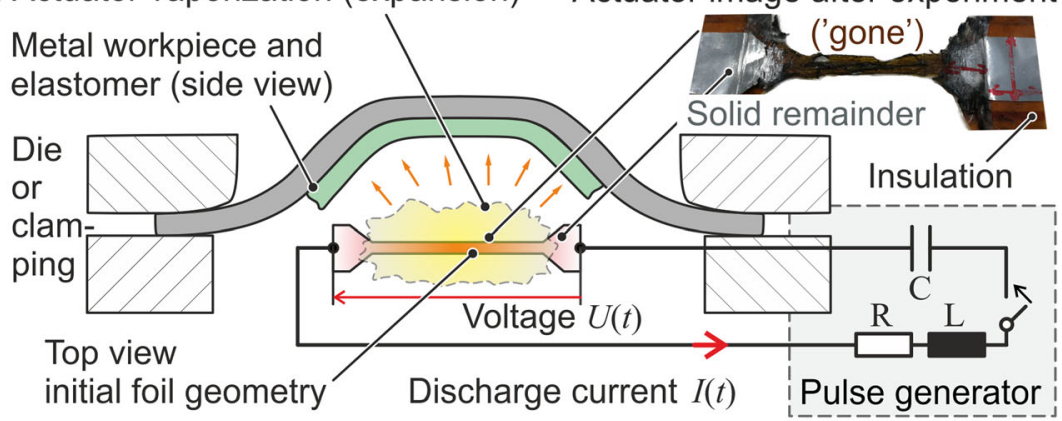

b)

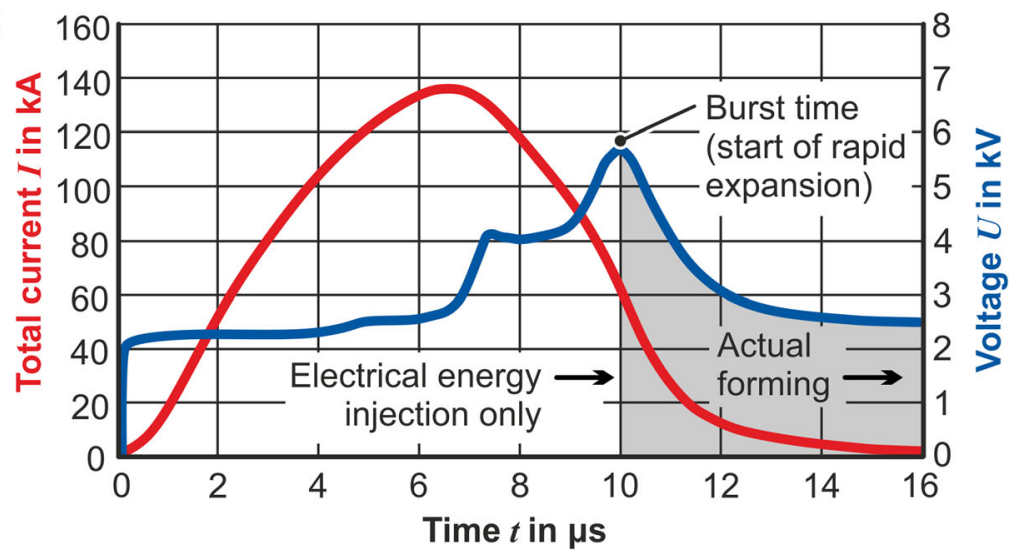

Vivek et al. [9] conducted a hybrid experiment by preforming a titanium sheet quasi-statically first, and the final cell case geometry was calibrated by VFAF subsequently to form fine features. The process parameters yielding the best appearing result were found after different experimental tryouts.

VFAF offers a high degree of flexibility (adjustment of capacitor bank, actuator design and placement, different clamping options for the blank, ...). Though, the process design for VFAF is still in the early stages. Many wasteful experiments often have to be performed in an experience-backed trial-and-error procedure to identify a proper setup configuration for a given forming task. This raises the need for a predictive method to ease and speed up the process design. The development and validation of such a method is the aim of this paper.

There is no established overall modeling and simulation procedure for VFAF yet. In ongoing research, Hahn et al. $[10]$ and Hahn and Tekkaya $[11,12]$ suggest basically two 'modeling levels':

1) A rather in-depth multi-physical level. There, the thermalelectric energy deposition as well as the forming through the interaction between the workpiece(s) and the expanding actuator are considered, with the help of a meshless method.

2) A reduced, more application-oriented and efficient level. In this case, the mechanical mesh- or 'chain'-based simulation step is treated separately. There, the (3D or 2D) forming of the blank initiated by an impulse assumed to be known in advance is modeled (e.g. deduced from an electrical circuit analysis).

In view of a practical process design, only the second level is followed in this paper.

\section{Predictive process design}

\section{Comparison with conventional sheet forming}

For many conventional sheet metal forming processes there is a well-known procedure or method for a predictive process design. For example, in classical quasi-static stamping or deep drawing the final part is defined by the matching positive punch and negative die, presuming no failure happens. The process is often displacement-controlled, so that it must -more or less- just be guaranteed that the maximum press force is greater than the resulting forming force. This can usually be modeled numerically in a comparatively straightforward manner. Another established example is the hydroforming process, which is inherently force/pressure-controlled. The constant and uniformly distributed fluid pressure required to form a certain die radius comes from the static equilibrium. The implementation is also comparatively simple because the pressure can be directly preset on the forming machine and - 
depending on the part complexity- even be predicted analytically, e.g. with the help of membrane theory.

In case of the energy-based process of vaporizing foil actuator forming, the procedure gets more elaborate as the vaporizing pressure is not only highly transient but also spatially varying. And it cannot be directly set on the forming machine, i.e. the capacitor bank. In addition, the modeling of the blank material becomes more demanding in high speed forming once the strain rate dependency becomes dominant. A locally varying pressure could be seen as a disadvantage, since it is likely to cause an inhomogeneous forming. But this can also be advantageous, since high short-term pressures are only generated where needed. This makes high clamping forces and therefore big machines expendable. Another general advantage of high speed forming is the quasi-removal of springback [13].

\section{Proposed procedure for vaporizing foil actuator forming (VFAF)}

Generally, a predictive process design for VFAF requires to successively answer the questions raised in Fig. 2. This introduces a two step-procedure: finding a proper forming taskspecific impulse distribution (I), and realizing this distribution electrically by vaporizing actuators (II).

\section{Mechanical optimization}

Theoretically, indefinitely many different impulse distributions would be possible. So, the first step poses an optimization problem. The optimization goal must be formulated so that the sheet displacements $u_{k}$ resulting from the forming ideally conform with the die geometry of the desired part. Since this geometry can entail many different targeted displacement values $u_{k T}$, a multi-objective optimization is pursued. The errors between the respective displacements present the objective functions to be minimized by varying the impulse load distribution $\boldsymbol{G}$ under the constraints of existing experimental limitations ('Exp'), i.e.

$\min _{\boldsymbol{G} \in \operatorname{Exp}}\left(\left|u_{1 T}-u_{1}\{\boldsymbol{G}\}\right|, \ldots,\left|u_{k T}-u_{k}\{\boldsymbol{G}\}\right|\right)$

(Fig. 3 a). Vaporizations develop in the narrowest cross sections of the actuators. A resultant 'experimental limitation' of a finite number $n$ of actuators in a setup is that each distinct vaporization area is supposed to be rectangular, with length $l$ and width $b$ (plus a smooth transition), to ensure a uniform Joule heating (Fig. 3 b). This yields a fairly uniform pressure amplitude $p_{b}$ at burst normal to the initial sheet plane ( $\left.x y\right)$ within each vaporization area, while the wider rest of the actuator remains practically pressureless. Different vaporization areas are not allowed to overlap, but their coordinate distances $d_{x}$ and $d_{y}$ may be varied in the foil plane (also $x y$ ) to adjust their positions (Fig. 3 b). So the free parameters, i.e. the virtually discrete burst pressure distribution (pressures, areas, positions), can be specified as

$\boldsymbol{G}=\left[\left(p_{b 1}, l_{1}, b_{1}, d_{x 1}, d_{y 1}\right), \ldots,\left(p_{b n}, l_{n}, b_{n}, d_{x n}, d_{y n}\right)\right]$.

The optimization task is to be performed on the basis of a finite element (FE) model for the dynamic forming simulation. For this, the model of Hahn and Tekkaya [11] is utilized. It is built in Abaqus/Explicit and mainly contains: the sheet blank using volume elements, the strain- and strain rate-dependent flow curve of the material (in this case the isotropic ZerilliArmstrong equation for DC01 steel), the part-specific die with a clamping, and a flexible load definition. For the latter, notionally setting the burst time to zero, the impulse (per unit area) representing the $n$th vaporization area, $P_{A n}$, is given by

$P_{A n}=\int_{0}^{t_{\text {end }}} p_{n}(t) \mathrm{d} t \cong \int_{0}^{\infty} p_{b n} \exp \left(\frac{-t}{t_{d n}}\right) \mathrm{d} t=p_{b n} t_{d n}$

where $t_{d n}$ is the decay time. While the pressure amplitude $p_{b n}$ can be modified in an experiment by changing the charging energy of the pulse generator, $W_{0}$, the decay time caused by the foil expansion is unknown a priori and not really adjustable in the experiment. Youngdahl [14] found that the final deformation result is virtually the same for different loadings of large effective amplitudes, meaning different combinations of $p_{b n}$ and $t_{d n}$ in the present case ( $p_{b n}>>$ static yield load), as long as the total impulse remains the same. Thus, if a global upper bound value is assumed for all decay times $t_{d}$, the correct impulse could be accomplished in the later experiment by simply increasing the charging energy. This is called a conservative approach, since a simulation having the same pressure amplitude as a related experiment implies a larger impulse then, so that less forming can be expected in the experiment owing to the actually shorter decay time. To establish an upper bound for $t_{d}$, the volumetric energy balance of a sheet element of thickness $s$ is considered. It can be stated that the
Fig. 2 Necessary procedure to establish a predictive process design

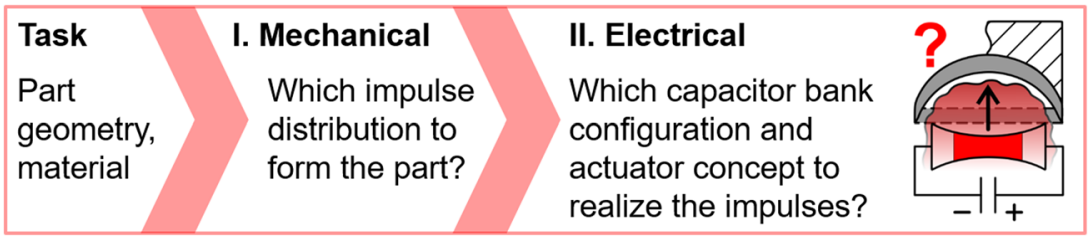


Fig. 3 a) Forming optimization (goal: $|\Delta u| \longrightarrow 0$ ), b) variable load distribution (red)

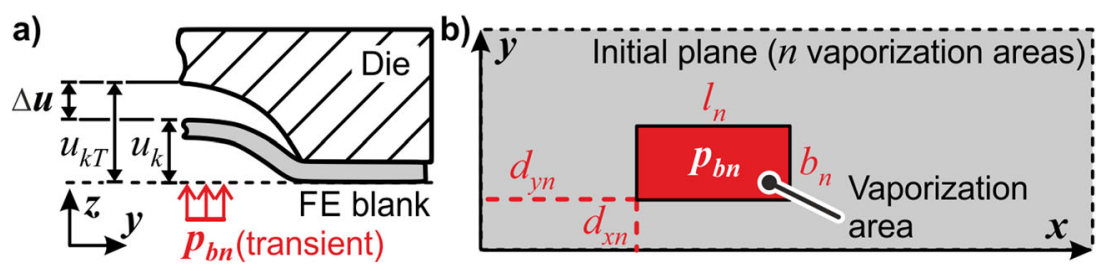

dominant energy components are just the kinetic energy $w_{\text {kin }}$ and the plastic energy $w_{p l}$, whose sum needs to equal the externally applied impulsive energy $w_{\text {ext }}\left(w_{\text {ext }} \approx w_{\text {kin }}+w_{p l}\right.$, see Fig. 4 which was confirmed by simulations). For the fast exponentially decaying pressure application with $\mathrm{d} w_{\text {ext }}=p /$ $s \cdot \mathrm{d} u$, the external work remains effectively constant $\left(w_{\text {ext,tot }}\right)$ with $p \approx 0$ after reaching the maximum kinetic energy $w_{\text {kin,max }}$. The kinetic energy is then continuously converted to plastic energy until the process end $t_{\text {end }}$. As both energy courses need to intersect at some point, $w_{\text {ext,tot }}=w_{\text {kin,max }}+w_{p l} *<w_{\text {kin,max }}+$ $w_{\text {ext,tot }} / 2$ must apply before (Fig. 4 ). With density $\rho_{0}$, maximum velocity $v_{\max }$, final displacement $u_{\text {end }}$, average pressure $\bar{p}$, and neglecting thickness changes, this inequality reads $w$ ext,tot $/ 2=\bar{p} u_{\text {end }} /(2 s)<w_{\text {kin, } \max }=\rho_{0} v_{\text {max }}^{2} / 2$. Inserting the impulse requirement $\bar{p} t_{\text {end }} \cong p_{b} t_{d}$ (cf. Eq. 3), after rearranging, gives

$t_{d}<\frac{\rho_{0} v_{\text {max }}^{2} t_{\text {end }} S}{p_{b} u_{\text {end }}}:=t_{d \text { Lim }}$.

From preliminary numerical and experimental investigations, which include i.a. laser-based velocity measurements $[11,12]$, the upper bound $t_{d \text { Lim }}$ from Eq. 4 was found to be ca. $2 \mu$ s for a $1 \mathrm{~mm}$ thick DC01 sheet. This value was used in all predictive simulations of the present work. If the total simulated impulse $P$, which is the sum of all impulses over their vaporization areas (left-hand side of Eq. 5), is associated with a certain experiment with known increased pressures $p_{b}^{\prime}$ (right-hand side of Eq. 5), the actual decay times $t_{d}^{\prime}$ may be

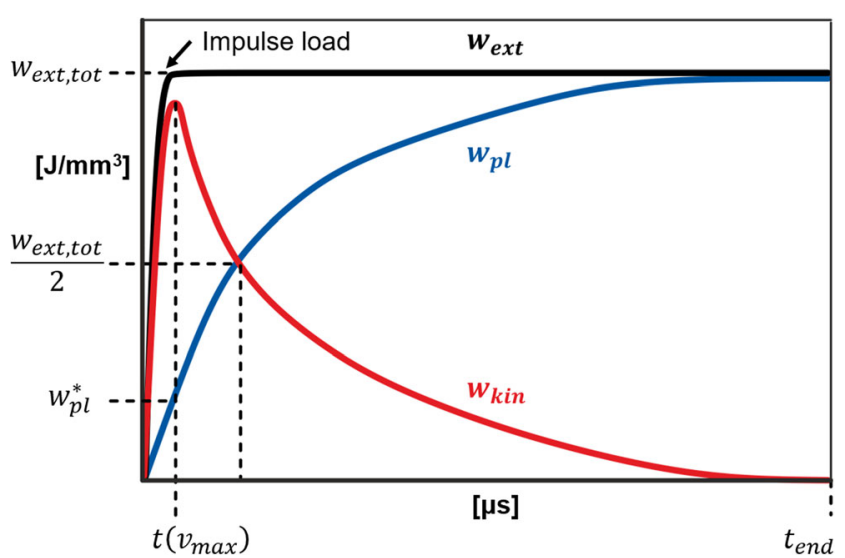

Fig. 4 Qualitative temporal energy evolution of a loaded sheet element (free forming) approximated retrospectively with the help of the impulse equivalence

$P=t_{d L i m} \sum_{i=1}^{n} p_{b i} l_{i} b_{i} \equiv \sum_{i=1}^{n} t_{d i}^{\prime} p_{b i}^{\prime} l_{i} b_{i}$.

A successful optimization as described above provides the burst impulse distribution $\boldsymbol{G}_{\text {goal }}$ required to form a desired part. Yet, the accompanying predicted pressure amplitudes are only valid for the introduced decay time $t_{d L i m}$, facilitating a conservative process design.

\section{Electrical realization strategy}

For every burst pressure amplitude $p_{b}$, a resistive energy deposition in the form of a burst energy density $w_{b}$ may be determined from the linear coupling relation $p_{b}\left(w_{b}\right)$ found by Grigoriev and Pavlenko [15]. This means that, besides all actuator widths $b$, lengths $l$, and positions from the mechanical optimization, all required energy depositions $w_{b}$ are already known for the second step in Fig. 2. The only remaining free process parameters to realize this energy deposition distribution are the total charging energy $W_{O}$ and the initial foil thicknesses $s_{f}$ of the vaporization regions.

The vaporization regions are interpreted as resistive cuboid circuit elements in a simplified $R L C$-analysis (resistance-inductance-capacitance). Particularly, the energy-dependence of the resistivity of the actuator material (pure aluminum) from the solid up to the gaseous state, $\rho_{r}[w]$, as well as the ratedependence of the possible energy deposition are taken into account. Such an analytical model, with average resistances $\bar{R}$ $=l \bar{\rho}_{r}\left[w_{b}\right] /\left(b s_{f}\right)$, is provided by Hahn et al. [16], supplemented by some recent data updates. ${ }^{1}$ In this model, the classical laws for series and parallel circuits are to be used (not explained here). The general procedure for the model is illustrated in Fig. 5. Finding admissible foil thicknesses for two vaporizations connected in series is relatively straightforward.

\footnotetext{
${ }^{1}$ The energy deposition law for aluminum foil actuators given in Hahn et al. [16], which must be fulfilled during the computations, was found to be more precise in the form:$$
w_{b}(l, \dot{\bar{w}})=13.5 \exp (-0.0664 l-3.8459) \dot{\bar{w}}^{(0.0027 l+0.2076)} \leq 2 w_{e v},
$$

with length $l$ in $\mathrm{mm}$, average energy deposition rate $\dot{\bar{w}}$ in $\mathrm{J} /(\mathrm{gs})$, and the full heat of evaporation $w_{e v} \cong 13.5 \mathrm{MJ} / \mathrm{kg}$. Then, $p_{b}\left(w_{b}\right) \cong 1.81 w_{b} / w_{e v}-0.48$ in GPa. Also, the eventually piecewise linear data $\rho_{r}[w]$ of Hahn et al. $[10]$ were incorporated. The other model equations are not repeated here.
} 
Fig. 5 Overall functioning of the electrical analytical model (inductance $L$ and capacitance $C$ of the setup are assumed constant)

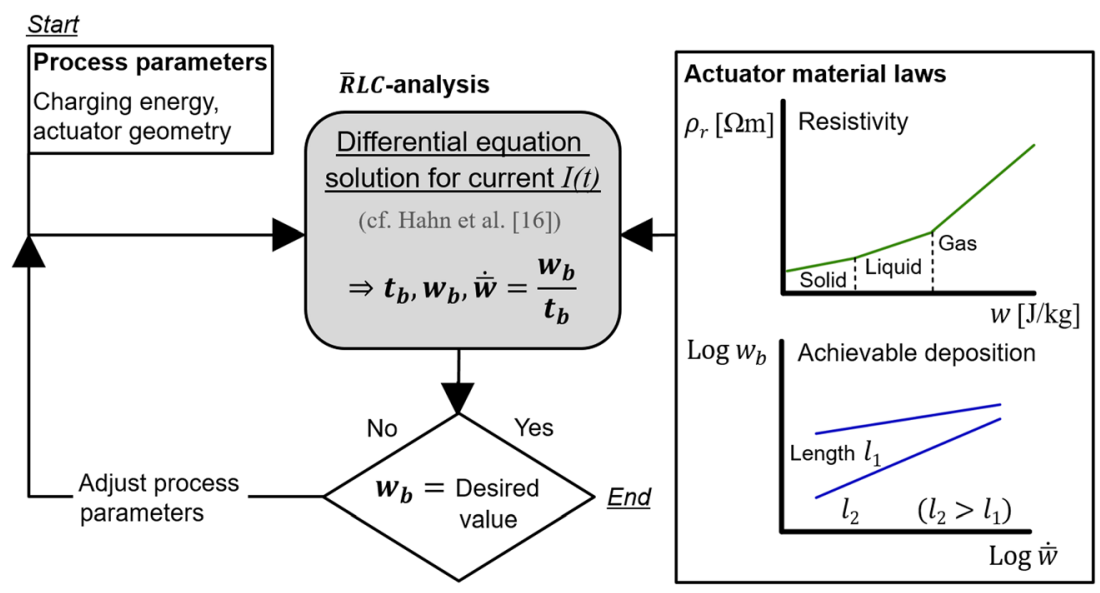

The reason is that the two total currents in both elements are inevitably the same when comparing the respective Joule heating equations for the required energy depositions. Whereas in a parallel vaporization connection, the current ratio is the invers ratio of the resistances. Since this applies independent of the voltage, some reference charging voltage (and thus energy) can be assumed first to find admissible foil thicknesses by separate model iterations of the parallel elements. When doing so, the first thickness can be chosen depending on market availability. Afterwards, the second thickness follows compulsorily. This way, the current ratio is determined by the separately identified thicknesses and the required resistivities. Every energy deposition distribution can finally be substituted by one equivalent resistance $R^{*}$. The mass of $R^{*}$ equates to the sum of the masses of all involved actuator elements. For a chosen capacitance $C$, inductance $L$, and charging energy $W_{0}$ of an existing pulse generator, the model then gives an estimation for the resulting total discharge current curve. Along with that comes an equivalent energy density at the burst time $t_{b}$ (which was purposefully set to zero in the mechanical simulations). Lastly, in the frame of additional model iterations, the charging energy may still need to be varied until the targeted equivalent energy deposition is met.

In summary, the process design procedure indicated in Fig. 2 can be reified as shown in Fig. 6.

\section{Theoretical result for an example part}

\section{Task definition}

The geometry displayed in Fig. 7 a was designed to serve as an example part. It mainly consists of two defined forming planes, named height 1 and 2, that are continuously connected by $4 \mathrm{~mm}$ radii while the outer walls have an inclination angle of $45^{\circ}$. The challenge in forming this part by vaporizing foil actuators is that the different heights and their joint basic dimensions $(85 \times 75 \mathrm{~mm})$ necessitate arranging preferably two different actuators in parallel ( $n=2$ in Eq. 2).

A rigid die that corresponds to the part geometry was incorporated into the numerical forming model. Due to the symmetry as seen in Fig. $7 \mathbf{b}$, the optimization parameters to be varied were defined as $\boldsymbol{G}=\left[p_{b 1}, l_{1}, b_{1}, d_{1}, p_{b 2}, l_{2}, b_{2}, d_{2}\right]$ within $85 \times 75 \mathrm{~mm}$. The impulse distribution therefore comprises 8 values to be found for attaining an optimum forming result ( 2 actuator widths and 2 lengths, their 2 distances from the $x$-axis, and the 2 pressure amplitudes). For the objective function definitions (cf. Eq. 1 and Fig. 3), 40 nodes lying in critical sections of the blank surface were selected (with respect to the fixed coordinate system in Fig. 7 b, 1 st section: symmetric $y z-$ plane, 2nd section: center of height 1 - parallel to the $x z$-plane, 3rd section: center of height 2 - parallel to the $x z$-plane, 4 th section: $x z$-plane). The targeted vertical $z$-displacements of those nodes match with the die geometry. The Isight software was chosen as optimization environment. I.a. the NSGA-II
Fig. 6 Summary of the proposed process design method

\begin{tabular}{|c|c|c|}
\hline Task & I. Mechanical & II. Electrical \\
\hline $\begin{array}{l}\text { Part } \\
\text { geometry } \\
\text { (die), } \\
\text { material } \\
\text { data }\end{array}$ & $\begin{array}{l}\text { Optimize } \\
\text { possible impulses } \\
\text { with FE model } \\
(\rightarrow \text { foil lengths, } \\
\text { widths, and } \\
\text { positions) }\end{array}$ & $\begin{array}{l}\text { Analytically } \\
\text { determine charging } \\
\text { energy and foil } \\
\text { thicknesses to } \\
\text { realize the found } \\
\text { impulses }\end{array}$ \\
\hline
\end{tabular}


Fig. 7 a) Targeted example part (dimensions in $\mathrm{mm}$ ), b) top view of the die including parameters to be determined (= vaporization areas and pressures in the initial plane)

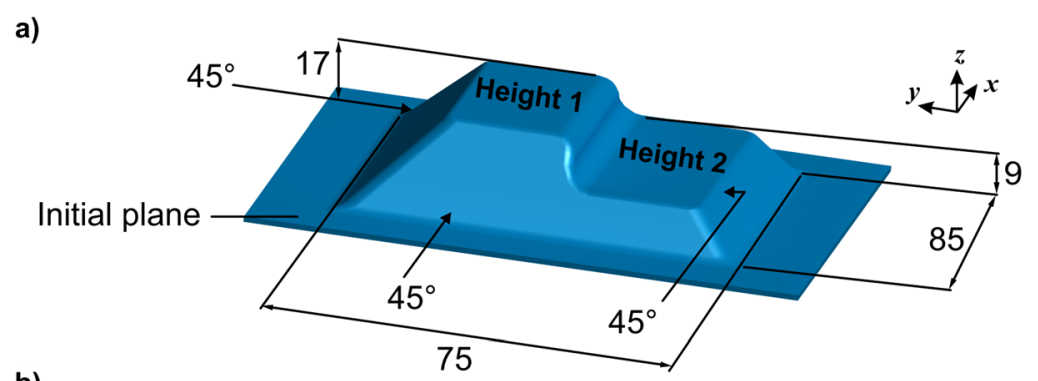

b)

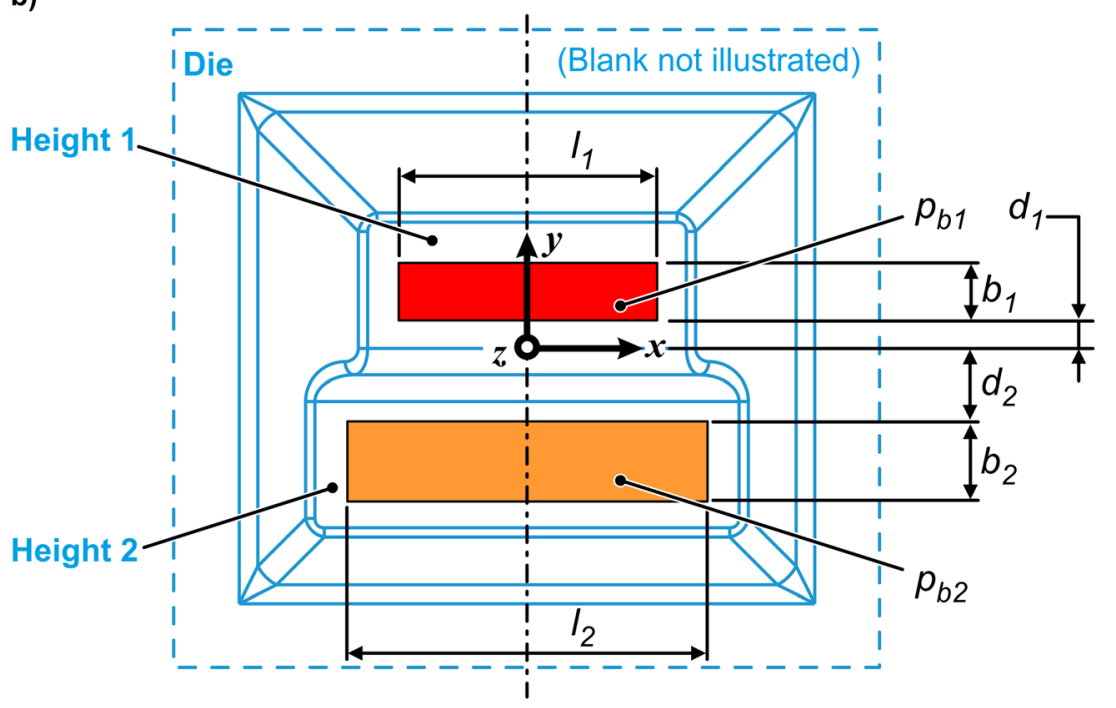

algorithm (see Van der Velden and Koch [17] for details), a standard choice for nonlinear multi-objective optimizations, was used to adopt Eq. 1 and $\mathbf{2}$ for the Abaqus model. The $120 \times 120 \times 1 \mathrm{~mm}$ DC01 blank was composed of ca. 400,000 elements. No mass scaling was used to correctly represent inertial forces, which are relevant for the displacement evolution in high speed forming. The total simulated forming process time was roughly $200 \mu \mathrm{s}$, as this was always beyond the point in time when the kinetic energy of the blank was fully dissipated plastically with no further alterations in the plastic energy.

\section{Optimization run}

During the optimization as described in the previous section, the impulse loading is changed and several simulations are conducted. In Fig. 8, some extracts from those simulations are compiled to qualitatively show the influence of the loading on the forming result. On the one hand, if the total impulses are too low, the forming is finished too early (Fig. 8 a). On the other hand, if the impulses are too high -or unfavourably positioned-, a bounce-back after hitting the die as well as local thinning can occur (Fig. 8 b). An acceptable forming in the sense of the optimization goal can be achieved in between (Fig. 8 c). Supplementing simulations further showed that friction as well as varied clamping forces only play a minor role for the final part geometry. Conducting the whole numerical optimization took about three days on a standard computer (CPU with 4 cores, $4.2 \mathrm{GHz}$ ).

The final optimization result is presented in Fig. 9, particularly for three sections where displacements of the objective functions lie. It can be seen that the die walls may not be expected to be completely filled in the optimized scenario. But at smaller, higher, or otherwise distributed impulses, the overall die filling would even be worse, similar to what is shown in Fig. 8 a and b. Especially the left-sided filling of height 1 and that of the transition from height 1 to height 2 in Fig. 9 b confirm the influence of inertia on the displacement evolution, as these blank regions are not directly subjected to a pressure but still formed. If arbitrarily distributed pressures were allowed in the optimization, which is not possible in reality, a more accurate die filling could probably be achieved. Nonetheless, the impulse distribution from Fig. 9 was taken as implementation goal for the experimental validation.

\section{Electrical calculation}

The strategy described in Section 2.2 was followed to electrically ensure the found twofold impulse distribution in a parallel $R L C$-circuit. The pulse generator to be used offers a capacitance of $360 \mu \mathrm{F}$. For the sake of energy and material efficiency, the minimum available foil thickness was selected 
a)
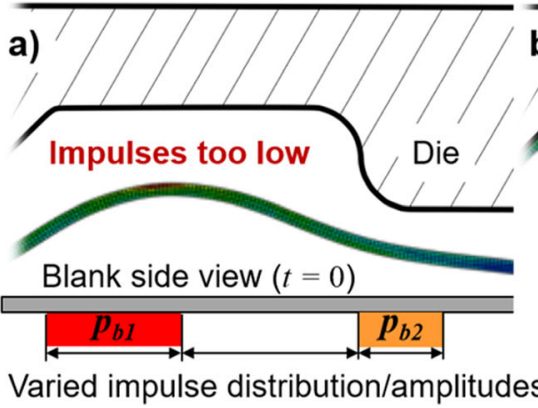

b)

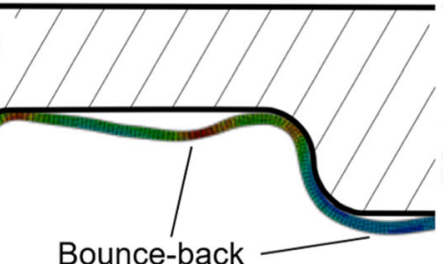

Bounce-back

and local thinning

Impulses too high c)

(Height 1)

$\approx$

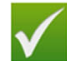

Acceptable impulses

Fig. 8 Simulation extracts from optimization run (a-c: symmetry plane section at process end, pressures initially acting perpendicular to the lower blank surface)

first for the vaporization area with the highest pressure amplitude. The required second thickness then followed, so that the consequent current ratio also assures the other, lower pressure amplitude at the same charging energy. The final charging energy $W_{0}$ was determined accordingly with the resulting equivalent resistance as part of the analytical modeling. All calculations were done in Matlab, they only took seconds. The entire process design result is compiled in Table 1. Only a part of the charging energy of the capacitor bank is injected into the actuators until they burst or vaporize. The total sodeposited resistive energy can be predicted based on the specific energy densities ( $w_{b}$-values in Table 1) integrated over the actuator volumes as $W_{\text {dep }, 0} \approx 2.55 \mathrm{~kJ}$. This means that roughly $40 \%$ of the suggested total energy input into the system, $W_{0}$, are expected to be deposited in the corresponding experiment. Yet, due to the conservative nature of the presented process design with respect to the pressure amplitudes, slightly higher charging energies and hence higher energy
Fig. 9 Final optimization result (Abaqus-Isight): $p_{b 1}=1.75 \mathrm{GPa}$, $p_{b 2}=0.42 \mathrm{GPa}, l_{l}=52 \mathrm{~mm}$, $l_{2}=80 \mathrm{~mm}, b_{1}=15 \mathrm{~mm}$, $b_{2}=22 \mathrm{~mm}, d_{1}=4 \mathrm{~mm}$, $d_{2}=15 \mathrm{~mm}$ (cf. Figure 7 b), a) 3D part view at process end, b) section $\mathrm{S} 1-\mathrm{S} 1, \mathrm{c}$ ) section $\mathrm{S} 2-\mathrm{S} 2, \mathrm{~d}$ ) section $\mathrm{S} 3-\mathrm{S} 3$

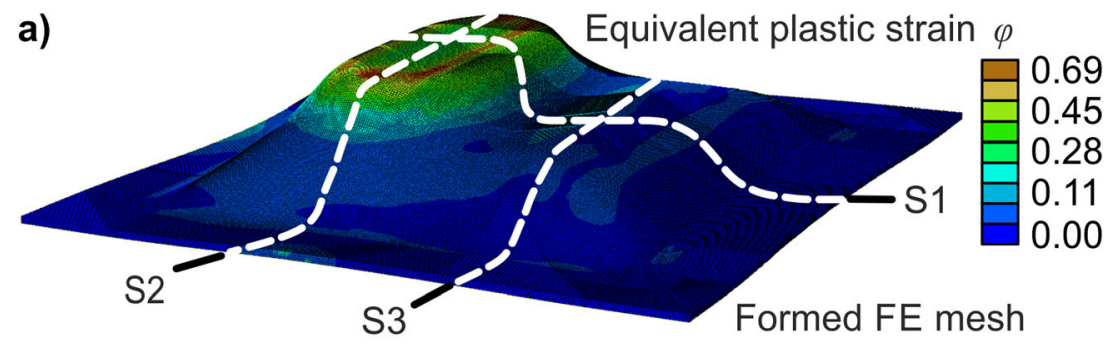

b)

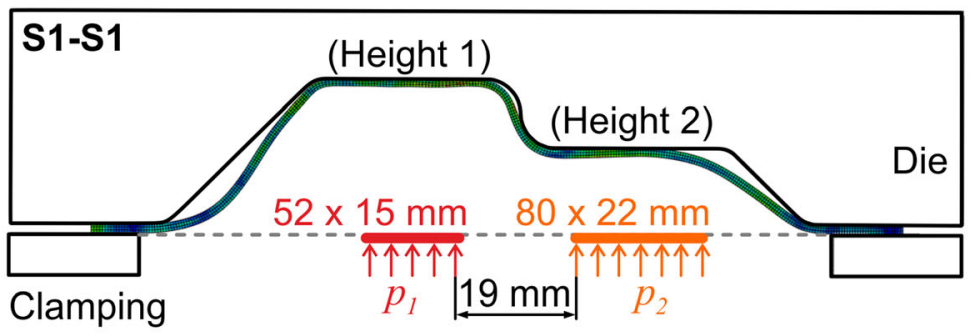

c)

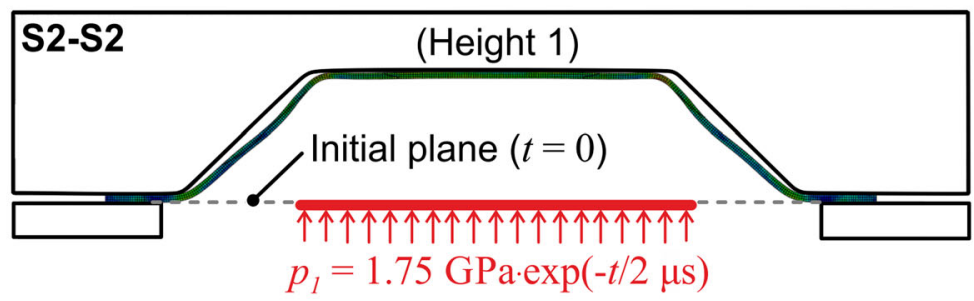

d) $\mathrm{S3-S3}$

(Height 2)

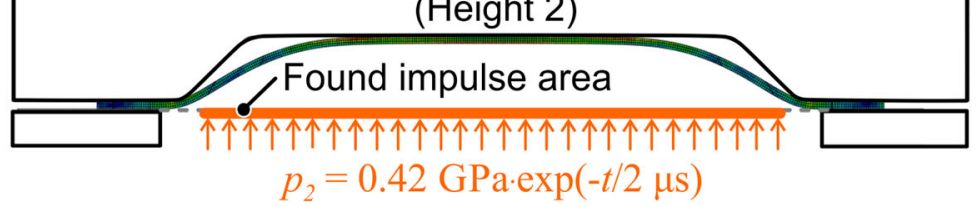


Table 1 Predicted mechanical-electrical process design result (referring to Figs. 7 / 9)

(19 mm distance between parallel areas 1 and 2) From numerical forming optimization, total impulse acc. to From analytical $R L C$-iterations Eq. 5: $P=4.2 \mathrm{kN} \cdot \mathrm{ms}$

\begin{tabular}{lllll} 
& Length $\boldsymbol{l}$ & Width $\boldsymbol{b}$ & Energy $\boldsymbol{w}_{\boldsymbol{b}}\left(\boldsymbol{p}_{\boldsymbol{b}}\right)$ & Foil thickness $\boldsymbol{s}_{\boldsymbol{f}}$ Energy $\boldsymbol{W}_{\boldsymbol{o}}$ \\
\hline Vaporization area 1 & $52 \mathrm{~mm}$ & $15 \mathrm{~mm}$ & $\begin{array}{l}16.65 \mathrm{MJ} / \mathrm{kg} \\
(1,750 \mathrm{MPa})\end{array}$ & $0.025 \mathrm{~mm}$ \\
Vaporization area 2 & $80 \mathrm{~mm}$ & $22 \mathrm{~mm}$ & $\begin{array}{l}7.07 \mathrm{MJ} / \mathrm{kg} \\
(420 \mathrm{MPa})\end{array}$ & $0.050 \mathrm{~mm}($ at $C=360 \mu \mathrm{F})$ \\
& & & & \\
\hline
\end{tabular}

depositions are expected to be needed for the forming, viz. to get the predicted total impulse listed in Table 1. The actuator dimensions and positions are fixed, though.

\section{Experimental validation}

\section{Experimental setup}

The derived data was implemented experimentally using the setup platform and pulse generator described in Hahn et al. [10] respectively in Hahn and Tekkaya [11, 12]. The main differences were that the waterjet-cut aluminum foils were arranged in parallel in this work for the first time, and that a sealed closed die was used. The die was machined from mild steel and contains a small drilled hole for pump-aided air evacuation. This is often needed in high speed forming, as a compressed air bubble between the sheet blank and the die could form and lead to unwanted counter-bulging. No lubrication was used. The time-dependent voltage $U(t)$ across the actuators was measured with a high-voltage divider at the electrodes, that are connected to the pulse generator, and the total current $I(t)$ of both actuators was measured with a Rogowski coil. These data were collected on a digital oscilloscope with a sampling rate of $2.5 \mathrm{GS} / \mathrm{s}$, and further processed in Matlab. The setup is depicted in Fig. 10.

\section{Results and discussion}

\section{Energy deposition consideration}

An analysis of the electrically deposited energy is carried out first. From the multiplication of the recorded current and voltage traces, ${ }^{2}$ the actually realized total energy deposition $W_{d e p}$ can be computed via integration until the burst time $t_{b}$, with the Joule heating concentrating in the vaporization regions. The recorded waveforms of the two parallel vaporizations were qualitatively hardly distinguishable from common single-foil experiments, giving a 'joint' $t_{b}$ of ca. $10 \mu \mathrm{s}$ at a

\footnotetext{
${ }^{2}$ (corrected by a term with a predetermined setup inductance of ca. $100 \mathrm{nH}$ ).
}

clear voltage peak (Fig. 1 b shows the waveforms of an experiment where the process parameters are consistent with Table 1). This behavior was also seen and pursued in the $R L C$ calculations during the process design procedure. The actuator's pressure regions were entirely vaporized or expanded in every experiment, as only the solid wider remainder of the foils survived. Compared to the predicted energy deposition $W_{\text {dep }, 0}, W_{\text {dep }}$ was measured to be $2.60 \mathrm{~kJ}$, confirming a satisfying agreement $\left(W_{\text {dep }} / W_{\text {dep }, 0} \approx 1.02\right)$. Charging energies higher than $W_{0}=6.3 \mathrm{~kJ}$ as in Table 1 were also tried. A moderate $20 \%$ increase in charging energy $\left(1.2 W_{0}\right)$ gave a measured deposition increase of about $27 \%$. A $30 \%$ higher charging energy $\left(1.3 W_{0}\right)$ already corresponded to an energy deposition $44 \%$ higher than $W_{\text {dep, } 0}$. This showcases the sensitivity of the only indirectly modifiable process parameter $w_{b}$. This improvement in electrical efficiency is also forecasted by the analytical model. Yet, some uncertainties must be noted. Even if an intended burst energy density $w_{b}$ is met, there is still a variance of about $7 \%$ in the pressure relation from Grigoriev and Pavlenko [15] on which the coupling between the electrical and the mechanical part of the presented modeling relies. In addition, the energy deposition law involved in the prediction of $w_{b}$ shows a standard deviation of about $14 \%$. Regarding the measured energy depositions themselves, up to ten repetitions per energy level pointed out a good reproducibility.

\section{Formed parts - Overview}

Like the mentioned energy depositions, the forming results proved to be very reproducible as well. Some formed parts are displayed in Fig. 11.

Generally, flange wrinkling was not a major issue in the experiments. On the left-hand side, the forming resulting from the initial process parameters listed in Table 1 is shown. The lower plane of the die, i.e. height 2, was just reached, while the higher die plane, i.e. height 1 , was not reached. This proves the earlier stated conservativeness of the process design. As an upper bound was set in the pressure optimization for the decay time, the actual decay time is shorter, giving a lower real impulse and thus an expectedly incomplete forming. A higher 
Fig. 10 Experimental setup a) at the beginning of assembly showing the actuators, b) completed - right before discharge with current flow along $x$-direction (plus a protecting box is placed on top of the whole setup)

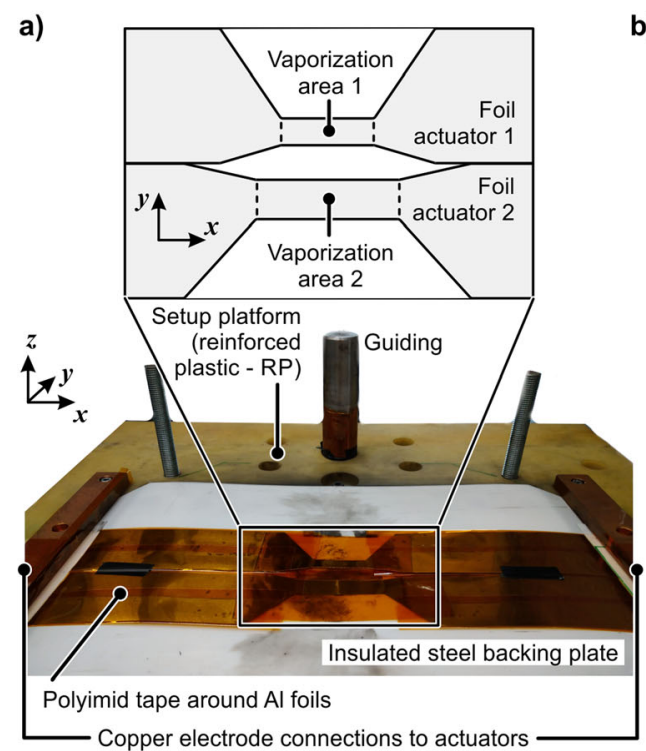

b) Section S1-S1 (not drawn to scale):
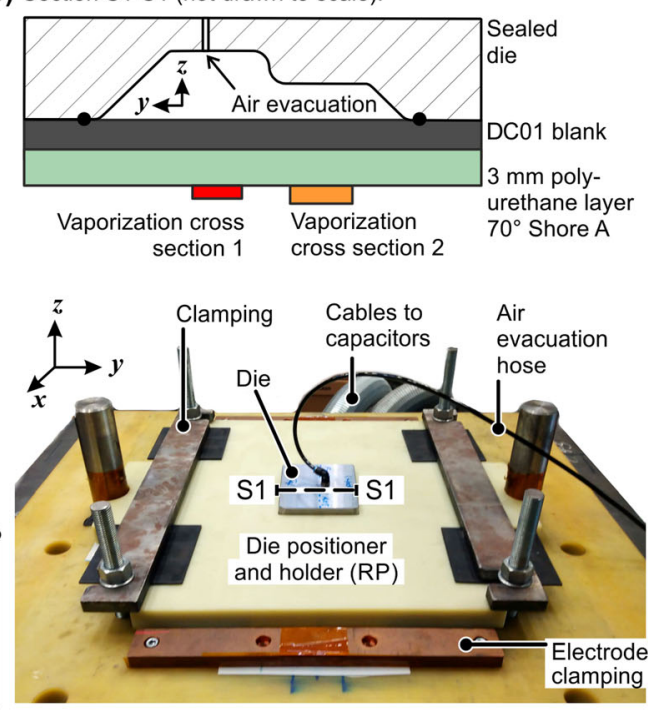

pressure amplitude generated by a higher charging energy can yield an impulse closer to the predicted one from Table 1. In case of the $20 \%$ charging energy increase $\left(1.2 W_{0}\right)$, in the middle of Fig. 11, plane 1 is almost reached while plane 2 is filled a little better compared to the first part. Increasing the initial charging energy by $30 \%\left(1.3 W_{0}\right)$ gave rise to cracking in the transition region between plane 1 and 2 , while both planes show sufficient filling (right-hand side, Fig. 11). Near this transition region, enhanced local thinning was also observable in large-impulse simulations. It can thus be concluded that the total impulse was probably already higher than that given in Table 1.

\section{Formed parts - Selected comparison}

To find the charging energy with noteworthy thinning but yet right before sheet failure, further experiments in the interval $\left[1.2 W_{0}, 1.3 W_{0}\right]$ were conducted, yielding a value of $1.24 W_{0}$. This value is considered the most suitable one, and the total impulse associated with it is consequently hypothesized to comply with the predicted one from Table $1, P \cong 4.2 \mathrm{kN} \cdot \mathrm{ms}$. The analytical model can also be used to count back the burst energy densities from a known total energy deposition. For $1.24 W_{0}$, where the total deposited energy was $3.43 \mathrm{~kJ}$, the pressure amplitudes can then be approximated as $p_{b 1}^{\prime}=$ $2.51 \mathrm{GPa}$ and $p_{b 2}^{\prime}=0.79 \mathrm{GPa}$ in conjunction with the mentioned relation $p_{b}\left(w_{b}\right)$. With this knowledge, and considering the impulse equivalence in Eq. 5, the used global decay time $t_{d L i m}=2 \mu$ s can be revised retrospectively as $t_{d 1}^{\prime}=1.40 \mu \mathrm{s}$ and $t_{d 2}^{\prime}=1.06 \mu \mathrm{s}$. This verifies that the process design with $t_{d L i m}$ is a robust and feasible approach. Because even if the upper bound decay time used in the simulations appears to be almost twice as high as one of the actual individual decay times, the needed change in charging energy to realize the so-predicted total impulse just amounts to $24 \%$. This is beneficial as not too many experiments are needed to find the optimum charging energy near the projected $W_{0}$.

The part geometry for $1.24 W_{0}$ was measured optically with the three-dimensional Atos system by GOM. In Fig. 12, the results are compared to the targeted and predicted geometry. No bounce-back effects were seen in both cases. For the observable geometric differences, there are two likely explanations:

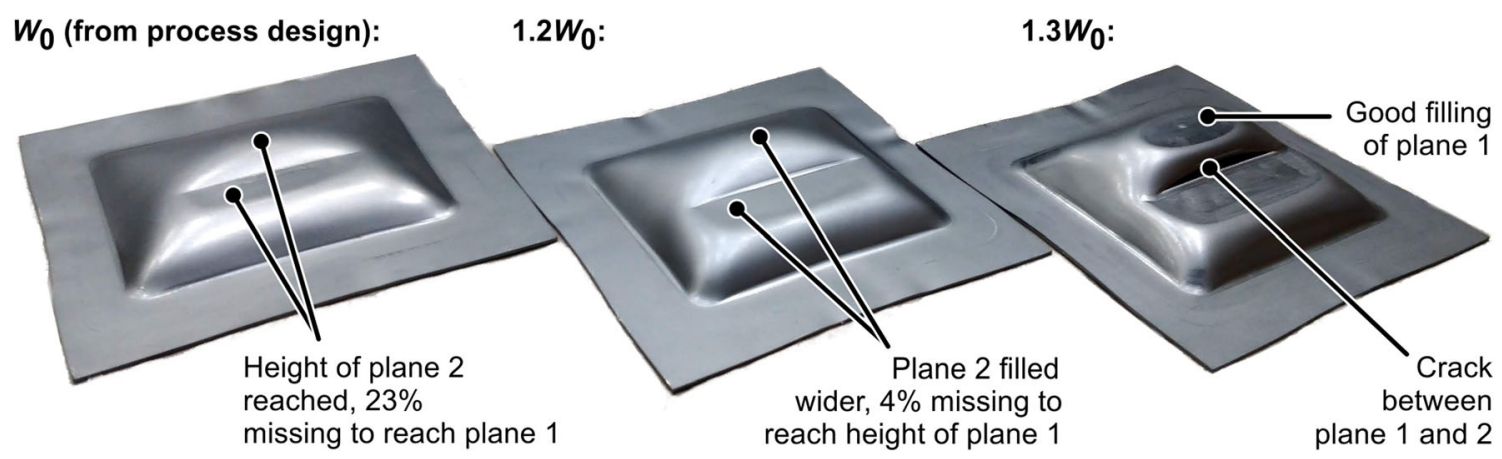

Fig. 11 Images of some experimental parts (initially $120 \times 120 \times 1 \mathrm{~mm} \mathrm{DC01}$ sheet blanks, charging energy $W_{0}$ according to Table 1 ) 


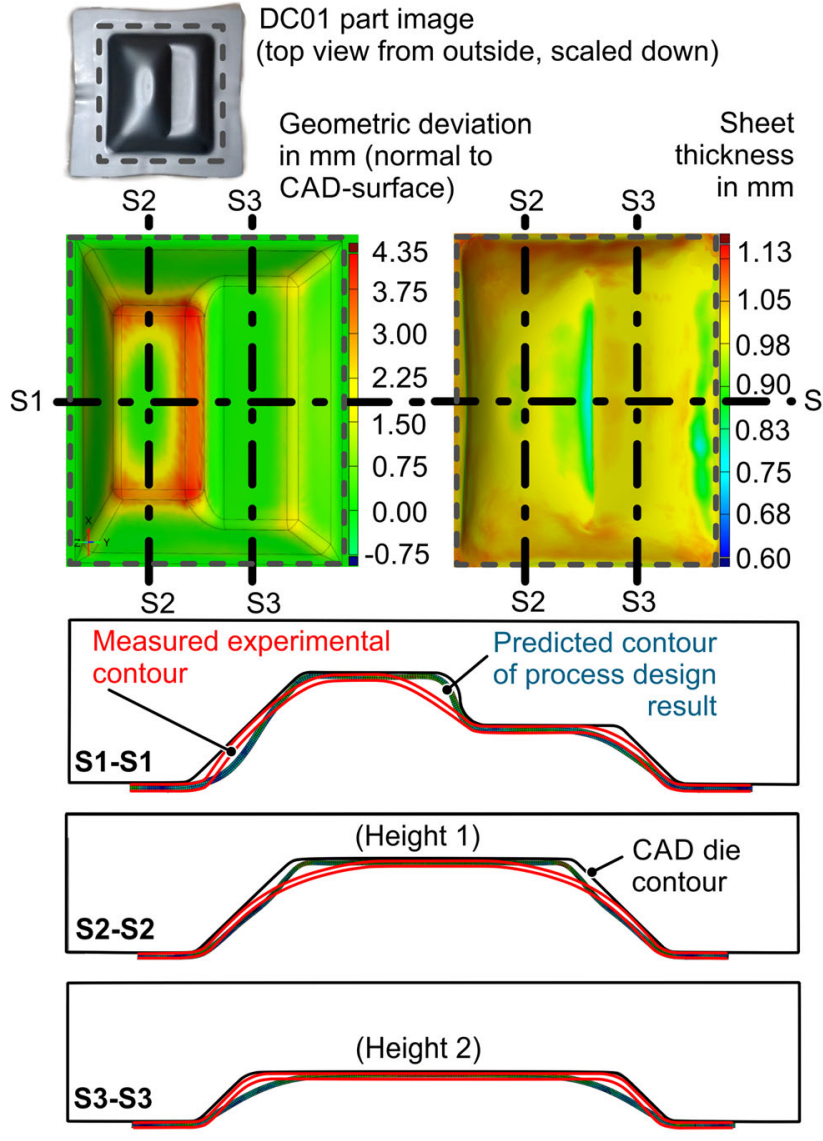

Fig. 12 Geometry comparison with the obtained results (top views and selected sections). Predicted total impulse: $4.2 \mathrm{kN} \cdot \mathrm{ms}$, experimental charging energy: $7.812 \mathrm{~kJ}$

First, the utilized FE modeling constitutes a reduction of the real problem, since the expanding actuator as well as the polyurethane interlayer are not taken into account. Instead, a predefined impulse loading is directly applied on the sheet. This seemed reasonable because the elastomer is not able to bear significant deformation forces due to its low stiffness compared to the metal blank. Preliminary numerical studies further showed too severe element distortions when trying to apply comparatively high vaporization pressures on an elastomer described by a hyperelastic material law, so it was excluded for the optimization simulations. On the one hand, as the clamped elastomer has a certain mass that can also be accelerated during the vaporization, it may still push the metal blank in the experiment. This may lead to regions in Fig. 12 where the experiment is closer to the targeted contour than the predicted outcome. On the other hand, the elastomer also features some damping property and therefore dissipates a fraction of the energy stemming from the input loading. So, less energy is there for the metal blank in the experiment, enabling some sort of pressure blurring. This, in turn, may explain the regions in Fig. 12 where the simulated result is closer to the targeted CAD (Computer Aided Design) contour of the die. In total, this gives an average geometric deviation between the predicted and the experimental upper sheet surface of ca. 5\% with a peak value of about $20 \%$ in the die corner regions.

Beside the consequences of the missing elastomer in the FE model, a second potential reason for deviations between experiment and simulation is the used flow curve. The rate-dependence of DC01 was approximated and extrapolated by the ZerilliArmstrong equation, supported by four different characterization tests at strain rates ranging from $2 \cdot 10^{-3}$ to $1.9 \cdot 10^{4} \mathrm{~s}^{-1}[11]$. The flow curve fitting posed inaccuracies of up to $10 \%$, while the accuracy at other process-relevant rates stays unknown.

Overall, given the necessary underlying simplifications of such a complex and highly dynamic multi-physics process, the demonstrated process modeling is considered acceptable. It allows for quickly and systematically finding relevant process parameters close to the optimum in advance. Nevertheless, some part geometries might not be formable solely using vaporizing actuators.

\section{Conclusions}

Previous research was extended and combined to provide a novel predictive method for the vaporizing foil actuator forming (VFAF) process design. Starting from the desired part geometry and material, the method first numerically yields an optimized part-individual impulse distribution that comes along with an electrical burst energy distribution. This distribution also determines the actuator designs in the blank plane. Employing electric circuit analytics, including a found energy deposition law, the aluminum foil thicknesses and charging energy are then determined for a given capacitor bank to ensure the inferred energy distribution. The method is conservative in terms of the pressure prediction, so that the desired forming should be realizable experimentally by slightly increasing the projected charging energy. It can be concluded that, despite some found process limitations concerning the geometric accuracy and remaining modeling challenges, a first experimental realization with parallelly vaporizing actuators for a part with different forming heights showed the general validity of the procedure. This ultimately lowers the number of required experimental try-outs, so that no pure trial-and-error procedure needs to be followed anymore. For certain customized sheet parts or prototypes of different alloys, quickly applying inexpensive consumable vaporizing actuators with the demonstrated process design may present an attractive choice.

Among others, $\mathrm{Yu}$ and Zheng [5] revealed an enhanced formability for particular steel sheets under high strain rates, like they also evolve in VFAF. The determination and inclusion of accompanying rate-dependent forming limit curves (FLC) in the process modeling would be an interesting field for future works. Such a failure criterion would add a safety aspect to the VFAF process design. 
Acknowledgements The authors thank Veerendra Kumar as a student assistant for his help in conducting the optimization and the experiments.

Code availability Not applicable.

Authors' contributions Conceptualization: Hahn $\mathrm{M}$ and Tekkaya AE; Methodology: Hahn M and Tekkaya AE; Validation: Hahn M; Formal analysis: Hahn M; Investigation (experimental/analytical/numerical): Hahn M; Writing - original draft preparation: Hahn M; Writingreview and editing: Hahn M and Tekkaya AE; Visualization: Hahn M; Supervision: Tekkaya AE; Project administration: Hahn M and Tekkaya AE; Funding acquisition: Hahn M and Tekkaya AE.

Funding Open Access funding enabled and organized by Projekt DEAL. This work was funded by the Deutsche Forschungsgemeinschaft DFG (German Research Foundation) in the frame of the project 391967465.

Data availability Data and material are not provided with this article.

\section{Declarations}

Conflict of interest The authors declare that they have no conflict of interest.

Open Access This article is licensed under a Creative Commons Attribution 4.0 International License, which permits use, sharing, adaptation, distribution and reproduction in any medium or format, as long as you give appropriate credit to the original author(s) and the source, provide a link to the Creative Commons licence, and indicate if changes were made. The images or other third party material in this article are included in the article's Creative Commons licence, unless indicated otherwise in a credit line to the material. If material is not included in the article's Creative Commons licence and your intended use is not permitted by statutory regulation or exceeds the permitted use, you will need to obtain permission directly from the copyright holder. To view a copy of this licence, visit http://creativecommons.org/licenses/by/4.0/.

\section{References}

1. Vivek A, Taber GA, Johnson JR, Woodward ST, Daehn GS (2013) Electrically driven plasma via vaporization of metallic conductors: a tool for impulse metal working. J Mater Process Technol 213(8): 1311-1326. https://doi.org/10.1016/j.jmatprotec.2013.02.010

2. Thurston BP, Vivek A, Nirudhoddi BSL, Daehn GS (2019) Vaporizing foil actuator welding. MRS Bull 44(8):637-642. https://doi.org/10.1557/mrs.2019.184

3. Nassiri A, Zhang S, Abke T, Vivek A, Kinsey B, Daehn GS (2017). Numerical modeling of high-velocity impact welding. In: Meyers $\mathrm{M}$ et al. (Eds) Proceedings of the 3rd Pan American materials congress. The Minerals, Metals \& Materials Series, Springer. https:// doi.org/10.1007/978-3-319-52132-9_9
4. Sow CT, Bazin G, Heuze T, Racineux G (2020) Electromagnetic flanging: from elementary geometries to aeronautical components. Int J Mater Form 13(3):423-443. https://doi.org/10.1007/s12289020-01547-y

5. Yu H, Zheng Q (2019) Forming limit diagram of DP600 steel sheets during electrohydraulic forming. Int J Adv Manuf Technol 104(1-4):743-756. https://doi.org/10.1007/s00170-019-03885-x

6. Vivek A, Daehn GS, Taber GA, Johnson JR (2013). Electrically driven rapidly vaporizing foils, wires and strips used for collision welding and sheet metal forming. Patent: US2013/0283878 A1

7. Vivek A, Daehn GS (2019). Vaporizing foil actuator configured as consumable tape. Patent: US2019/0061045 A1

8. Cai S (2015). Tailored and double-direction pressure distributions for vaporizing-foil forming. Dissertation, TU Dortmund

9. Vivek A, Brune RC, Hansen SR, Daehn GS (2014) Vaporizing foil actuator used for impulse forming and embossing of titanium and aluminum alloys. J Mater Process Technol 214(4):865-875. https:// doi.org/10.1016/j.jmatprotec.2013.12.003

10. Hahn M, Goyal S, Gies S, Tekkaya AE (2019). Numerical modeling of energy deposition for vaporizing foil actuator forming. In: proceedings of the 13th international conference on numerical methods in industrial forming processes - NUMIFORM, Portsmouth (NH), USA

11. Hahn M, Tekkaya AE (2020). Experimental and numerical analysis of the influence of burst pressure distribution on rapid free sheet forming by vaporizing foil actuators. Metals 10 (6), 845 - special issue on numerical and experimental advances in innovative manufacturing processes. https://doi.org/10.3390/met10060845

12. Hahn M, Tekkaya AE (2020) A quick model for demonstrating high speed forming capabilities. Mech Res Commun 108:103579. https://doi.org/10.1016/j.mechrescom.2020.103579

13. Daehn GS, Altynova M, Balanethiram VS, Fenton G, Padmanabhan M, Tamhane A, Winnard E (1995) High-velocity metal forming - an old technology addresses new problems. J Minerals Metals Mat Soc 47(7):42-45. https://doi.org/10.1007/ BF03221230

14. Youngdahl CK (1970). Correlation parameters for eliminating the effect of pulse shape on dynamic plastic deformation. Journal of Applied Mechanics, Transactions ASME, pp. 744-752. https://doi. org/10.1115/1.3408605

15. Grigoriev AN, Pavlenko AV (2009) Pressure generated by the electric explosion of metal foils. Tech Phys Lett 35(9):865-868. https:// doi.org/10.1134/S1063785009090235

16. Hahn M, Hansen SR, Gies S, Vivek A, Daehn GS, Tekkaya AE (2018). Prediction of achievable energy deposition for vaporizing foil actuators. In: proceedings of the 8th international conference on high speed forming - ICHSF, Columbus (OH), USA. https://doi. org/10.17877/DE290R-19132

17. Van der Velden A, Koch P (2010). Isight design optimization methodologies. ASM Handbook 22B, ASM International

Publisher's note Springer Nature remains neutral with regard to jurisdictional claims in published maps and institutional affiliations. 